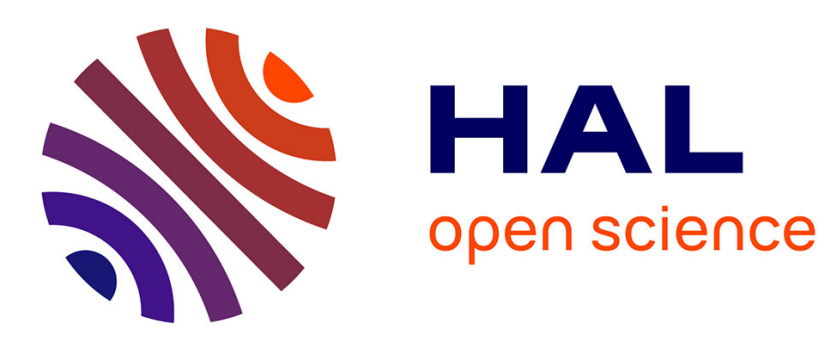

\title{
NEW RESULTS ON WEAK INTERACTIONS - SUMMARY AND ASSESSMENT
}

\author{
L. Sehgal
}

\section{To cite this version:}

L. Sehgal. NEW RESULTS ON WEAK INTERACTIONS - SUMMARY AND ASSESSMENT. Journal de Physique Colloques, 1982, 43 (C3), pp.C3-22-C3-31. 10.1051/jphyscol:1982302 . jpa-00221855

\section{HAL Id: jpa-00221855 https://hal.science/jpa-00221855}

Submitted on 1 Jan 1982

HAL is a multi-disciplinary open access archive for the deposit and dissemination of scientific research documents, whether they are published or not. The documents may come from teaching and research institutions in France or abroad, or from public or private research centers.
L'archive ouverte pluridisciplinaire HAL, est destinée au dépôt et à la diffusion de documents scientifiques de niveau recherche, publiés ou non, émanant des établissements d'enseignement et de recherche français ou étrangers, des laboratoires publics ou privés. 
JOURNAL DE PHYSIQUE

Colzoque C3, supplément au no12, Tome 43, décembre 1982

NEW RESULTS ON WEAK INTERACTIONS - SUMMARY AND ASSESSMENT

\author{
L.M. Sehga1 \\ III. Physikalisches Institut, Technische Hochschule, Fhysikzentmum, \\ 5100 Aachen, F.R.G.
}

New results have been presented at this Conference that have been obtained from a variety of lepton-hadron processes (neutrino interactions, muon scattering, atomic parity violation and beam dump experiments). I shall attempt in this summary to place these results in the context of the general endeavour to probe and define the phenomenological properties of the weak interactions.

\title{
1. NEUTRAL CURRENTS
}

\subsection{Weak-Electromagnetic Interference in $\mu \mathrm{N}$ Interactions}

The NA4 experiment at CERN (BCDMS collaboration) ${ }^{1}$ ) has compared the inclusive cross section of $\mu^{+}$and $\mu^{-}$on a Carbon target:

$$
\mu^{ \pm} \mathrm{C} \rightarrow \mu^{ \pm} \mathrm{X}
$$

It is found that

$$
\sigma\left(\mu^{+}, \lambda\right) \not \sigma\left(\mu^{-},-\lambda\right)
$$

where $\lambda(-\lambda)$ denote the polarizations of the $\mu^{+}\left(\mu^{-}\right)$beams. The beams actually used were nearly left-handed $\mu^{+}$and nearly right-handed $\mu^{-}$, with $|\lambda|=0.8$ and $E_{\mu} \approx 200 \mathrm{GeV}$. The asymmetry $(2)$ is a violation of the one-photon exchange approximation, which demands equal $\mu^{+}$and $\mu^{-}$cross sections, regardless of polarization.

A charge asymmetry of the type (2) can arise from two sources:

$$
B=\left[\sigma\left(\mu^{+}\right)-\sigma\left(\mu^{-}\right)\right] /\left[\sigma\left(\mu^{+}\right)+\sigma\left(\mu^{-}\right)\right]=B_{\text {rad }}+B_{\text {weak }} .
$$

The first is a QED radiative correction that can be symbolically represented as

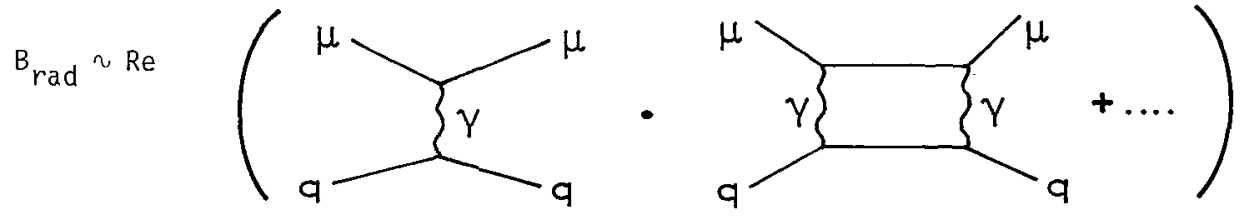


while the second denotes a weak-electromagnetic interference effect

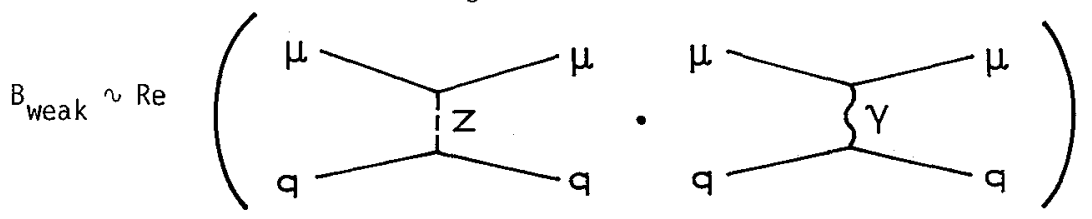

The radiative effect $B_{\text {rad }}$ has been calculated by Bardin and Shumeiko. ${ }^{2)}$ The weak asymmetry $B_{\text {weak }}$ depends on the neutral current interaction of muons and quarks. Writing the effective Lagrangian as

$$
\begin{aligned}
\mathscr{L}_{\text {eff }}(\mu q)=-\frac{G}{\sqrt{2}} & {\left[\bar{\mu}_{\alpha} \mu\left\{\varepsilon_{V V}(q) \bar{q} \gamma_{\alpha} q+\varepsilon_{V A}(q) \bar{q} \gamma_{\alpha} \gamma_{5} q\right\}\right.} \\
& \left.+\bar{\mu} \gamma_{\alpha} \gamma_{5} \mu\left\{\varepsilon_{A V} \bar{q} \gamma_{\alpha} q+\varepsilon_{A A}(q) \bar{q} \gamma_{\alpha} \gamma_{5} q\right\}\right]
\end{aligned}
$$

the weak asymmetry is

$$
\begin{gathered}
\left.B_{\text {weak }}=K\left[|\lambda| \in \frac{2}{3} \varepsilon_{V A}(u)-\frac{1}{3} \varepsilon_{V A}(d)\right\}-\left\{\frac{2}{3} \varepsilon_{A A}(u)-\frac{1}{3} \varepsilon_{A A}(d)\right\}\right], \\
K=-\left(\sqrt{2} G / e^{2}\right)(9 / 5)\left[\frac{1-(1-y)^{2}}{1+(1-y)^{2}} Q^{2}\right] \frac{q-\bar{q}}{q+\bar{q}}
\end{gathered}
$$

The last factor in $\mathrm{K}$ is a dilution factor due to antiquarks, close to unity for the conditions of this experiment. One therefore expects $B_{\text {weak }}$ to be proportional to the variable $w \equiv Q^{2}\left[1-(1-y)^{2}\right] /\left[1+(1-y)^{2}\right]$, a prediction borne out by the data. The slope of this variation yields the combination of weak interaction parameters

$$
|\lambda|\left\{\frac{2}{3} \varepsilon_{V A}(u)-\frac{1}{3} \varepsilon_{V A}(d)\right\}-\left\{\frac{2}{3} \varepsilon_{A A}(u)-\frac{1}{3} \varepsilon_{A A}(d)\right\}=0.45 \pm .11_{-.07}^{+.05}
$$

with $|\lambda|=0.8$. Note that the measured quantity is a linear combination of a parity-conserving and a parity-violating term. By contrast, the SLAC $\vec{e} D$ experiment ${ }^{3)}$ measured a purely parity-violating effect, summarized by ${ }^{4)}$

$$
\left\{\frac{2}{3} \varepsilon_{A V}(u)-\frac{1}{3} \varepsilon_{A V}(d)\right\}+0.25\left\{\frac{2}{3} \varepsilon_{V A}(u)-\frac{1}{3} \varepsilon_{V A}(d)\right\}=-0.26 \pm .015 .
$$

The constraints (6) and (7) are illustrated in Figs. 1a and 1b.

In the standard $S U(2) \times U(1)$ theory, the parameters appearing in (6) have the values

$$
\begin{aligned}
& \frac{2}{3} \varepsilon_{V A}(u)-\frac{1}{3} \varepsilon_{V A}(d)=-\frac{1}{2}+2 \sin ^{2} \theta \\
& \frac{2}{3} \varepsilon_{A A}(u)-\frac{1}{3} \varepsilon_{A A}(d)=-\frac{1}{2}
\end{aligned}
$$


C3-24

JOURNAL DE PHYSIQUE

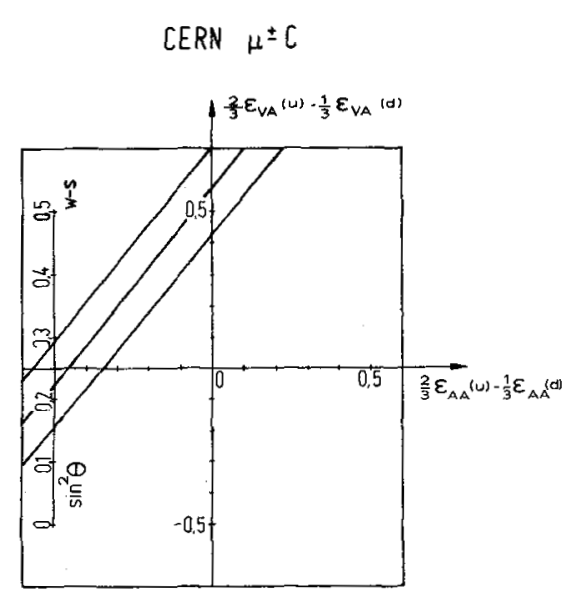

Fig. Ia: Constraints on weak muon-quark couplings imposed by charge asymmetry in $\mu^{*}$-Carbon scattering
(BCDMS collaboration, Ref. 1).

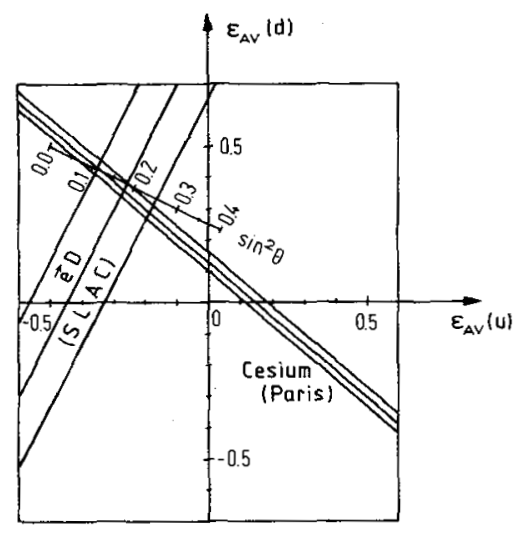

Fig. 2: Constraint on electron-quark couplings imposed by parity violation measurement in Cesium
(Ref. 5) and SLAC ex D experiment (Ref. 3).
SLAT Er D

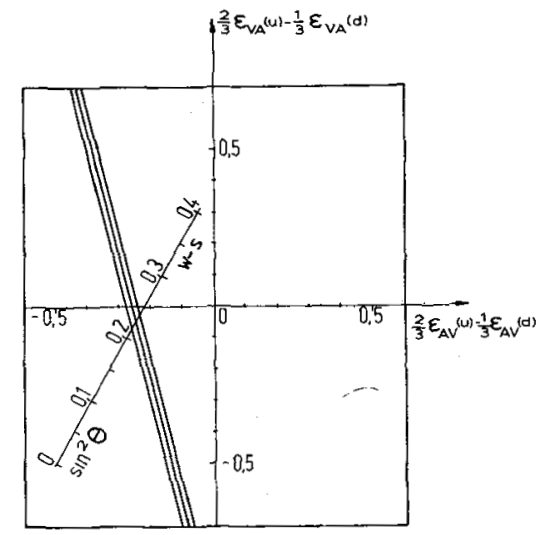

Fig. 16: Constraints on weak electron-quark couplings
imposed by SLAC polarization asymmetry (REf. 3 ).

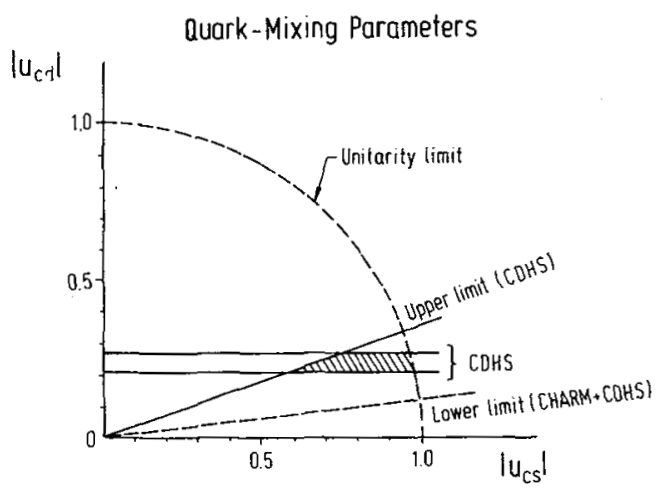

Fig. 3: Mixing parameters of the charmed quark derived 
The measured asymmetry determines

$$
\sin ^{2} \theta=0.22 \pm .06+.05
$$

In a slightly more general context in which the $\mu_{\mathrm{R}}^{-}$is allowed to have a non-zero isospin $I_{3 R}(\mu)$, the expressions in (7) are replaced by $-1 / 2+I_{3 R}(\mu)+2 \sin ^{2} \Theta$ and $-1 / 2-I_{3 R}(\mu)$ respectively. Taking $\sin ^{2} \theta=0.23$, the data give

$$
I_{3 R}(\mu)=0.01 \pm 0.06_{-0.03}^{+0.04}
$$

consistent with the standard assumption of right-handed singlets.

It is amusing to note that the weak parameters given in (8) coincide with the coupling constants $C_{V}$ and $C_{A}$ which occur in a completely different neutral current sector, namely $\nu_{\mu}$ e scattering. This is a reflection of the factorization property inherent in a 11 single- $Z$ models, combined with the specific quantum number assignment $I_{3 L}=\left(\frac{1}{2},-\frac{1}{2}, \frac{1}{2},-\frac{1}{2}\right)$ for $\left(\nu_{\mu}, e, u, d\right)$ in the standard theory.

\subsection{Parity Violation in CS Atom}

An experiment by Bouchiat et al. ${ }^{5)}$ has observed a parity-violating effect in the scattering of circularly polarized light from Cesium vapour. An interference effect is seen between a parity-violating amplitude $E_{1}^{P V}$ and a known parity-conserving Stark-induced amplitude $B E_{0}$, with the result

$$
\operatorname{ImE}_{1}^{\mathrm{PV}} / \beta=-1.34 \pm .22 \pm .11 \mathrm{mV} / \mathrm{cm}
$$

This constitutes a striking $6 \sigma$ effect with only $8 \%$ systematic uncertainty. The theoretical expectation for $\sin ^{2} \Theta=0.219 \pm .014$ is stated to be $-1.73 \pm .07 \pm 10 \%$; the last uncertainty being due to atomic physics. In terms of the parameters of the neutral current Lagrangian (4) the quantity measured is

$$
Q_{W}=-2\left[\varepsilon_{A V}(u)(2 Z+N)+\varepsilon_{A V}(d)(Z+2 N)\right] \text {. }
$$

My assessment of the above result is that $\left[Q_{W}\right]^{C S}=-55.2 \pm 11.5$, yie]ding the constraint

$$
\varepsilon_{A V}(u)+1.12 \varepsilon_{A V}(d)=0.147 \pm .031 .
$$

It is interesting to note that this combination is almost orthogonal to the one determined by the "intercept" measured in the SLAC $\overrightarrow{\mathrm{e}} \mathrm{D}$ experiment ${ }^{3)}$ :

$$
\left[2 \varepsilon_{A V}(u)-\varepsilon_{A V}(d)\right] / 3=0.30 \pm .08 \text {. }
$$

The constraints (13) and (14) are illustrated in Fig. 2. 


\subsection{Results from Neutrino Physics}

There has been refinement as well as diversification in the study of neutral currents in neutrino processes. Some of the new results are as follows.

(i) Diffractive $\rho^{0}$ Production. An experiment at FNAL ${ }^{6)}$ reports the observation of diffractive $\rho^{0}$ production by neutral currents: $\bar{\nu}+N \rightarrow \bar{v}+N+\rho^{0}$. Comparing with the charged current process $\bar{v}+\mathrm{N} \rightarrow \mu^{+}+N+\rho^{-}$, and using vector meson dominance, it is possible to determine the parameter ' $\alpha$ ' of the neutral hadron current ${ }^{4)}$ :

$$
\sigma\left(\bar{v} N \rightarrow \bar{v} \rho^{0} N\right) / \sigma\left(\bar{v} N \rightarrow \mu^{+} \rho-N\right)=\frac{1}{2} \alpha^{2}
$$

The result is $\alpha=0.44 \pm 0.18$. (Recall that in the standard model $\alpha=1-2 \sin ^{2} \theta$.)

(ii) Coherent $\pi^{\circ}$ Production: The Aachen-Padova experiment has observed a signal for the coherent process $\nu_{\mu}+A 7^{27} \rightarrow \nu_{\mu}+A I^{27}+\pi^{\circ}$. 7) This reaction is largely determined by the divergence of the axial isovector current, and so selectively determines the parameter $\beta$. A preliminary estimate yields

$$
\beta=1.3 \pm 0.67
$$

where the error includes a large uncertainty from theory. The result is compatible with the standard expectation $\beta=1$.

(iii) Single $\pi$ Production: A new analys is of old measurements of single pion production has been made by Fog $i^{8}$ ), with a view to extracting the isoscalar couplings $\gamma$ and $\delta$. The results are

$$
\begin{array}{r}
\gamma=-0.202+0.077 \\
-0.123 \\
\delta=0.07+.103 \\
-.102
\end{array}
$$

to be compared with the standard prediction $\gamma=-2 \sin ^{2} \theta / 3, \delta=0$.

(iv) Inclusive Scattering off Protons and Neutrons: Recent experiments in deuterium bubble chambers have measured the cross section ratio $\sigma^{N C} / \sigma^{C C}$ on protons and neutrons separately. The results, converted into the chiral couplings of $u$ and $d$ quarks, are
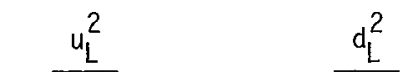
$0.19 \pm .06$
$0.13 \pm .04$
(15', Ref. 9)
$0.14 \pm .04$
$0.18 \pm .05$
(BEBC, Ref. 10). 
The standard theory prediction $\left|d_{L}\right|>\left|u_{L}\right|$ is not yet decisively demonstrated by the above measurements. The right-handed couplings $u_{R}$ and $d_{R}$ remain, as before, poorly resolved.

(v) Couplings of strange quarks: In a comparative study of the y-distribution of CC and NC interactions, the CHARM experiment ${ }^{1 \hat{1})}$ has detected a contribution from the NC coupling of strange quarks present in the $s \bar{s}$ sea. The coupling strength, when referred to that of the d-quark is

$$
\left(s_{L}^{2}+s_{R}^{2}\right) /\left(d_{L}^{2}+d_{R}^{2}\right)=1.39 \pm 0.43
$$

consistent with GIM symmetry, which predicts unity.

(vi) Couplings of charmed quarks: The CDHS experiment has observed the diffractive process $v+N \rightarrow v+N+\psi .^{12}$ ) Comparing the cross section with that of $\mu+N \rightarrow \mu+N+\psi$, and assuming vector and axial vector currents to contribute with equal weight (as in the Z-givon fusion model) one arrives at an estimate of the NC coupling strength of charmed quarks

$$
\left(c_{L}^{2}+c_{R}^{2}\right) /\left(u_{L}^{2}+u_{R}^{2}\right)=2.1 \pm 1.0 \text {, }
$$

compatible with unity. It remains to be understood why a fusion mechanism, involving the emission of a coloured quantum from the nucleus, should be relevant to a diffractive channel in which the target suffers little or no excitation.

(vii) Neutrino-Electron Scattering: A new measurement of the ratio of $v_{\mu} e$ and $\bar{v}_{\mu} e$ cross sections has been reported by the CHARM experiment. ${ }^{13)}$ This ratio delivers a value of $\sin ^{2} \Theta$ relatively free of normalization errors:

$$
\sin ^{2} \theta=0.215 \pm .040 \text { (stat.) }+0.015 \text { (syst.) }
$$

Combined with the earlier measurement of $\sigma\left(\bar{\nu}_{\mu} e\right)$, the new result also gives a measure of the overall strength parameter $\rho$ of the NC Lagrangian,

$$
\rho=1.12 \pm 0.12 \text { (stat.) } \pm 0.11 \text { (syst.) }
$$

in agreement with the value $\rho=1$ in the standard mode1.

\section{CHARGED CURRENTS}

\subsection{Quark Mixing Parameters from $\mu^{+} \mu^{-}$data}

The CDHS experiment has made a detailed study of $\nu$ - and $\bar{\nu}$-induced $\mu^{+} \mu^{-}$events originating from the production and decay of charmed particles. ${ }^{14)}$ The objective is to determine the strength of the $|\Delta C|=1$ cross section and thereby deduce the quark mixing parameters $U_{c d}$ and $U_{c S}$. In a world containing $u, c, d, s$ quarks only, 
these parameters would simply be $U_{c d}=\sin \theta_{C}, U_{C S}=\cos \Theta_{C}, \theta_{c}$ being the Cabibbo angle; but given the existence of at least three quark doublets, these parameters must be considered unknown, subject only to the unitarity constraint

$$
\left|u_{c d}\right|^{2}+\left|u_{c s}\right|^{2}+\left|u_{c b}\right|^{2}+\ldots=1 \text {. }
$$

The inclusive cross sections for $\left(_{\nu}\right)+N \rightarrow \mu^{-}+\mu^{+}+X$ at asymptotic energies, assuming complete acceptance, are

$$
\begin{aligned}
& \frac{d \sigma(2 \mu)}{d x d y}=\frac{G^{2} M E}{\pi}\left[\left|U_{c d}\right|^{2} q+\left|U_{c s}\right|^{2} q_{S}\right] B_{\mu} \\
& \frac{d \sigma(2 \mu)}{d x d y}=\frac{G^{2} M E}{\pi}\left[\left|U_{c d}\right|^{2} \bar{q}+\left|U_{c S}\right|^{2} q_{S}\right]_{B}
\end{aligned}
$$

where $q=x(u+d), \bar{q}=x(\bar{u}+\bar{d}), q_{s}=x(2 s)$, in familiar notation, and $B_{\mu}$ is the average branching ratio of the charmed particle mixture produced in these reactions. Comparing with the single muon cross sections

$$
\begin{aligned}
& \frac{d \sigma(1 \mu)}{d x d y}=\frac{G^{2} M E}{\pi}\left[\left(\left|U_{u d}\right|^{2}+\left|U_{c d}\right|^{2}\right) q+\left(\left|u_{u s}\right|^{2}+\left|U_{c s}\right|^{2}\right) q_{s}+\bar{q}(1-y)^{2}\right] \\
& \frac{d \bar{\sigma}(1 \mu)}{d x d y}=\frac{G^{2} M E}{\pi}\left[q(1-y)^{2}+\left(\left|U_{u d}\right|^{2}+\left|u_{c d}\right|^{2}\right) \bar{q}+\left(\left|u_{u s}\right|^{2}+\left|U_{c s}\right|^{2}\right) q_{s}\right]
\end{aligned}
$$

one can, in principle, determine $U_{c d}$ and $U_{c s}$. In practice, this is achieved only after a careful correction of the dimuon cross sections for effects of threshold and acceptance.

Following this procedure, the CDHS experiment ${ }^{14)}$ finds

$$
\begin{aligned}
& \left|u_{c d}\right|=0.24 \pm .03 \\
& \left|U_{c d}\right| /\left|U_{c s}\right|=(0.33 \pm 0.03)[2 S /(\bar{U}+\bar{D})]^{1 / 2} .
\end{aligned}
$$

The latter result involves the ratio of strange to non-strange sea contributions to the nucleon momentum. A safe upper 1 imit is $2 S /(\bar{U}+\bar{D})<1$, while a rough lower 1 imit $2 S /(\bar{U}+\bar{D})>0.16$ is obtained by combining the CDHS measurement of $\bar{U}+\bar{D}$ with the NC y-distribution measured by CHARM. ${ }^{11)}$ The resulting constraint is

$$
0.12<\left|\frac{U_{c d}}{U_{c s}}\right|<0.36
$$

The allowed doma in of $U_{c d}$ and $U_{c S}$ is $i 1 l$ ustrated in Fig. 3. 


\subsection{Limits on right-handed currents}

The $y$-distribution of the reaction $\bar{v}+N \rightarrow \mu^{+}+X$ has been analysed in the framework of a Lagrangian containing both $V-A$ and $V+A$ currents:

$$
\mathcal{L}_{C C}=-(G / \sqrt{2}) \bar{\mu}_{\alpha}\left(1+\gamma_{5}\right) \nu\left[J_{\alpha}(V-A)+\rho J_{\alpha}(V+A)\right] \text {. }
$$

The CDHS experiment obtains the limit $\left.{ }^{15}\right)$

$$
\rho^{2}<0.009
$$

Likewise, the chiral structure of the charmed current has been analysed by studying the $y$-distribution of $\mu^{+} \mu^{-}$events. Assuming the interaction to have the form

$$
\mathcal{L}_{C C}^{\Delta C=1}=-(G / \sqrt{2}) \vec{\mu}_{\alpha}\left(1+\gamma_{5}\right) \cup\left[g_{L} J_{\alpha}^{\Delta C=1}(V-A)+g_{R} J_{\alpha}^{\Delta C=1}(V+A)\right],
$$

two experiments have obtained the results

$$
\begin{aligned}
g_{R}^{2} /\left(g_{L}^{2}+g_{R}^{2}\right) & =0.15 \pm 0.10 \quad(\text { CHARM, Ref. 16) } \\
& <0.07(95 \% \text { C.L.) (CDHS, Ref. 14). }
\end{aligned}
$$

\section{SEARCHES FOR ANOMALOUS BEHAVIOUR}

\subsection{Flavour-Changing Neutral Currents}

An experiment at FNAL (CCFRS collaboration) ${ }^{17}$ ) has studied prompt $\mu$ production in a beam dump, and has searched for $\mu^{+} \mu^{+}$and $\mu^{-} \mu^{-}$events among a sample of dimuons. At $90 \%$ confidence, an upper 1 imit

$$
\frac{\mu^{-} \mu^{-}+\mu^{+} \mu^{+}}{\mu^{-} \mu^{+}}<0.8 \%
$$

is obtained, which, if interpreted in terms of possible $D^{0}-\bar{D}^{0}$ mixing gives

$$
P\left(D^{\circ}+\bar{D}^{\circ}\right)<4.4 \%,
$$

thus constraining a possible $\Delta C=1$ neutral current.

\subsection{Scalar Couplings}

The possibility that scalar quanta play a role in weak interactions prompts a continued search for scalar couplings in lepton-hadron processes. One signature is a $y^{2}$ term in the $y$-distribution of neutrino reactions. The CHARM experiment ${ }^{11}$ ) 
reports 1 imits

$$
\begin{aligned}
g_{S, P}^{2} / g_{V, A}^{2} & <0.06 \text { for charged currents } \\
& <0.03 \text { for neutral currents }
\end{aligned}
$$

Another limit may be obtained by noting that scalar NC interactions, which flip he] icjty, cannot contribute to weak-electromagnetic interference in electronquark scattering but can affect neutrino-quark processes. The fact that

$$
\left\langle\sin ^{2} \theta\right\rangle_{v q} \approx\left\langle\sin ^{2} \theta\right\rangle_{e q}
$$

suggests that such couplings are sma11. Note that if $\left\langle\sin ^{2} \theta\right\rangle$ vq is derived from a comparison of NC and CC reactions through a Paschos-Wolfenstein type relation ${ }^{18)}$

$$
\frac{1}{2}-\left\langle\sin ^{2} \Theta\right\rangle_{\nu q}=\frac{\sigma(\nu \rightarrow \nu)-\sigma(\bar{\nu} \rightarrow \bar{\nu})}{\sigma\left(\nu \rightarrow \mu^{-}\right)-\sigma\left(\bar{\nu} \rightarrow \mu^{+}\right)} \text {, }
$$

such a derivation is insensitive to the existence of scalar CC or NC couplings, since any scalar contributions cancel out in the numerator as well as denominator of (34).

\section{3 e- $\mu$ Universal ity}

The validity of e- $\mu$ universality in charm-decay has come under question ${ }^{19)}$ in connection with the CERN beam dump "puzzle", namely e/ $\simeq 0.6 \pm 0.2$. A new experiment at FNAL ${ }^{20)}$ yields a ratio $1.0 \pm 0.3$, compatible with universality. Any suggestion of a difference in the $e$ - and $\mu$-branching ratios of charmed particles must contend with the nearly equal rate (within $10 \%$ ) of $\mu^{-} \mu^{+}$and $\mu^{-} e^{+}$events in neutrino interactions ${ }^{14)}$. Smal 1 departures of $e / \mu$ from unity could arise from radiative corrections that produce differences in the $\nu_{e}$ and $\nu_{\mu}$ spectra of charm decay products.

\subsection{Compos ite Quarks?}

One signature for composite quarks is a non-zero radius. Experiments on $\mathrm{e}^{+} \mathrm{e}^{-} \rightarrow \mathrm{q} \bar{q}$ 1 imit the electromagnetic radius to $R<(100 \mathrm{GeV})^{-1}$. Another manifestation could be that the weak charged current vertex is not $\gamma_{\alpha}\left(1+\gamma_{5}\right)$ but $\gamma_{\alpha}\left(1+g_{A} \gamma_{5}\right)$. It is natural to expect that this renormalisation of the axial coupling would be very sma11, i.e. $\left|1-g_{A}\right| \approx m_{q} R \approx 10^{-4}$. However, a direct analysis of the charged current data yields on $7 y$ the weak constraint

$$
\left|\frac{1-g_{a}}{1+g_{a}}\right|<0.1 \text {. }
$$




\section{References}

1. ARGENTO, A. et a1. (BCDMS collaboration; presented by A. STAUDE).

2. BARDIN, D. and SHUMEIKo, N., Sov. J. Nuc1. Phys. 29 (4) (1979) 499.

3. PRESCOTT, C. et al., Phys. Lett. 77B (1979) 524.

4. HUNG, P. and SAKURA I, J., Ann. Rev. Nuc. Part. Sci. 31 (1981) 375.

5. BOUCHIAT, M., GUENA, J., HUNTER, L. and POTTIER, L. (Contributed paper, presented by L. POTTIER).

6. AMNOSOV, V. et a1. (IHEP-ITEP-FNAL collaboration; contributed paper 0303).

7. FAISSNER, H., Proc. XVI Rencontre de Moriond at Les Arcs (1981), Ed. TRAN THANH VAN; NIEBERGALL, F., CERN-EP/82-105 (1982), to be published in Proceedings Neutrino' 82 Conference.

8. FOGLI, G., Bari preprint, to be published in Nucl. Phys. B.

9. KAFKA, T. ét al., Phys. Rev. Lett. 48 (1982) 910.

10. Amsterdam-Bergen-Bologne-Padova-Pisa-Saclay-Torino collaboration, paper presented at Neutrino ' 82 Conference.

11. JONKER, M. et a1. (CHARM collaboration), Phys. Lett. 102B (1981) 67; BóSSER, F., Proc. Neutrino ' 81 (Maui), Eds. R. CENCE et al., Vol. I, p. 328.

12. ABRAMOWICZ, H. et a). (CDHS collaboration), Phys. Lett. 109B (1981) 115.

13. BERGSMA, F. et a1. (CHARM collaboration), CERN-EP/82-109 (1982), submitted to Phys. Lett.

14. ABRAMOWICZ, H. et al (CDHS collaboration), contributed paper 0318 , CERN-EP/82-77 (1982), submitted to Z. für Physik; KLEINKNECHT, K., Dortmund preprint UNIDO-82/272, to be published in Proc. Neutrino ' 82 Conference.

15. ABRAMOWICZ, H. et aT. (CDHS collaboration), Z. für Physik 12C (1982) 225; contributed paper 0705.

16. JONKER, M. et a1. (CHARM collaboration), Phys. Lett. (1982)

17. CCFRS collaboration (paper presented by A. BODEK).

18. PASCHOS, E. and WOLFENSTEIN, L., Phys. Rev. D7 (1973) 91.

19. BARGER, V. et a1., Hawa i preprint UH-511-465-82 (1982).

20. BALL, R. et a1. (experiment E-613 at FNAL, presented by B. ROE). 